\title{
Remotely sensed habitat diversity predicts butterfly species richness and community similarity in Canada
}

\author{
Jeremy T. Kerr*†, T. R. E. Southwood ${ }^{\ddagger}$, and Josef Cihlar* \\ *Canada Centre for Remote Sensing, Natural Resources Canada, 588 Booth Street, Ottawa, ON, Canada K1A 0Y7; and ₹Department of Zoology, \\ University of Oxford, South Parks Road, Oxford OX1 3PS, United Kingdom \\ Contributed by T. R. E. Southwood, July 30, 2001
}

\begin{abstract}
Although there is no shortage of potential explanations for the large-scale patterns of biological diversity, the hypothesis that energy-related factors are the primary determinants is perhaps most extensively supported, especially in cold-temperate regions. By using unusually high-resolution biodiversity and environmental data that have not previously been available, we demonstrate that habitat heterogeneity, as measured by remotely sensed land cover variation, explains Canadian butterfly richness better than any energy-related variable we measured across spatial scales. Although species-richness predictability declines with progressively smaller quadrat sizes, as expected, we demonstrate that most variability $(>90 \%)$ in butterfly richness may be explained by habitat heterogeneity with secondary contributions from climatic energy. We also find that patterns of community similarity across Canada are strongly related to patterns of habitat composition but not to differences in energy-related factors. Energy should still be considered significant but its main role may be through its effects on within-habitat diversity and perhaps, indirectly, on the sorts of habitats that may be found in a region. Effects of sampling intensity and spatial autocorrelation do not alter our findings.
\end{abstract}

S patial variability in species richness is a common phenomenon at different spatial scales and extents and has formed the basis for innumerable ecological and evolutionary studies (e.g., refs. 1-5). The best known-and widely studied-example of a species-richness gradient is the so-called "latitudinal gradient," the tendency for the richness of most taxa to increase toward the tropics (6). Many studies have attempted to identify a single factor as being globally responsible for this large-scale pattern, but recent work suggests that there may be a series of factors that play different roles within broad geographic regions $(3,7)$. Given the increasing importance of climate change and other humaninduced environmental changes, the conservation biological importance of understanding contemporary biodiversity patterns is substantial.

There is general consensus that within cold or temperate terrestrial regions, the most important predictors of species richness are related to heat- or energy-related aspects of climate, such as potential evapotranspiration (PET) or solar radiation (8-10). Monotonic strong relationships between energy and richness have been observed for all vertebrate $(3,5)$ and many invertebrate $(9,11)$ and plant assemblages $(8)$. Heat-related measurements of energy may influence regional species totals through the aggregated effects on individual species physiology or some other mechanism (2). Net primary productivity (NPP) generally relates very strongly to both heat-related aspects of climate and to species richness but NPP-richness correlations are most variable in warmer regions. The mechanisms that give rise to correlations between NPP or climate, respectively, and species richness are fundamentally different: NPP represents a resourcebased hypothesis (plant productivity may be partitioned between species) whereas climate does not.

To date, most large-scale tests of diversity hypotheses have used relatively coarse atlas data to measure biophysical variables (e.g., refs. 3-5 and 7-9). Although such manual interpretative methods may inflate errors, they seem to provide reasonable estimates of climate (12). Other factors, such as physical habitat measurements, are not so easily estimated from paper maps. In particular, habitat heterogeneity measurements can predict species richness over broad geographical areas by using large quadrats $(3,4)$.

Remotely sensed detection of land cover is likely to improve on alternative methods of measuring habitat heterogeneity. Satellite imagery, when processed and classified by using highquality algorithms, is capable of detecting habitats that are actually present (13). Land cover classification is a very active area of research (14), and quite detailed classifications based on data from the most advanced sensors (e.g., Spot4/Vegetation; ref. 15) exist for many areas, including Canada, the United States, and Western Europe. Remote sensing has received much attention partly because it refines existing methods of generating biophysical measurements $(12,16)$. Such measurements improve on previous methods of detecting the relationship between environment and species richness. However, topographic relief remains the most commonly used measurement of habitat heterogeneity (e.g., refs. 3 and 4). Elevation data estimate habitat heterogeneity coarsely, at best, and assume that areas of low relief have low habitat diversity. Topography makes no predictions about spatial variability in species richness in the absence of mountain ranges. Furthermore, the relationship between topography and habitat heterogeneity is static, whereas remotely sensed measurements of habitat heterogeneity are dynamic and are capable of incorporating other factors that influence species distributions, such as disturbances (e.g., fire). There has been little effort to use such high-resolution direct estimators of habitat heterogeneity to predict species richness and none that compares such predictions with those based on species richness-energy theory.

Climate is commonly observed to predict species richness better than topography-based measurements of habitat heterogeneity (e.g., ref. 5). Assuming both factors actually play a role in this issue, energy and heterogeneity should relate to species richness differently. Numbers of Amazonian amphibian species increase with heterogeneity created by topographic boundaries, leading to greater beta diversity (17). High habitat heterogeneity that has persisted through long periods correlates with regional tree species richness in Asia, potentially through higher speciation rates (18). However, Lepidopteran species richness increases with PET within individual biomes independently of habitat heterogeneity measures (19). Both factors may therefore play independent and strong roles in determining diversity. The habitat heterogeneity-species richness relationship has an intuitive mechanistic basis: few species are found in all habitats, thus the addition of new habitats to a sample should lead to an increase in regional species diversity totals. Therefore, habitat

Abbreviations: NPP, net primary productivity; PET, potential evapotranspiration; AVHRR, advanced very high-resolution radiometer; VGT, VEGETATION; SA, spatial autocorrelation. †To whom reprint requests should be addressed. E-mail: jeremy.kerr@ccrs.nrcan.gc.ca.

The publication costs of this article were defrayed in part by page charge payment. This article must therefore be hereby marked "advertisement" in accordance with 18 U.S.C. $\S 1734$ solely to indicate this fact. 
heterogeneity probably relates to regional diversity through species turnover (beta diversity). The species richness-energy hypothesis does not consider beta diversity explicitly; instead, it suggests that within-habitat diversity should rise with local energy availability $(2,20,21)$. However, if greater energy availability is associated with higher species turnover, we predict that differences in energy availability between areas should inversely correlate with measurements of their biotic similarity (i.e., large differences in climate should lead lower community similarity). Energy and habitat heterogeneity may contribute independently to predict regional species-richness patterns through influences on diversity within (energy) and between (habitat heterogeneity) habitats. High species turnover (high beta diversity or low community similarity) may "feed back" to increase local species richness through mass effects, but this effect may be undetectable across large regions.

In this study, we investigate butterfly species-richness patterns in Canada, an area where the richness-energy hypothesis is expected to make stronger predictions than competing hypotheses $(1-3,5,8,11)$. We employ pioneering high-resolution biodiversity and environmental data. Beta diversity has rarely been integrated into studies of regional diversity patterns; therefore, an additional study objective is to examine butterfly community structure across Canada and its relationship to energy and heterogeneity. High-resolution measurements of habitat heterogeneity should predict community similarity if this factor does relate to diversity through its effect on beta diversity. Similarly, if energy controls richness through effects on species turnover, we predict that differences in energy availability between quadrats will relate to community similarity measurements.

\section{Methods}

Sampling System and Data Sources. Three grid systems covering Canada's complete geographical extent were constructed and are of progressively larger quadrat sizes. Two of the grid systems consist of equal area quadrats of 2- and 5-degree latitudinal extents, respectively, and variable longitudes. The third grid system is that used most frequently in biodiversity studies in North America and consists of quadrats that are $2.5^{\circ} \times 2.5^{\circ}$ south of $50^{\circ} \mathrm{N}$, and $2.5^{\circ}$ (latitude) $\times 5^{\circ}$ north of $50^{\circ} \mathrm{N}$. No grid system, regardless of base quadrat size, is truly equal area if coastal regions are included. Consequently, the area of every quadrat in all grids was calculated and used as a covariate throughout statistical analyses. It was not significant in any model. Geographic analyses were conducted by using geographic information systems (GIS), including ARC/INFO GRID and IDRISI 32, and the image analysis package, $\mathrm{PCI}$. GIS scripts, $\mathrm{C}++$ programs, and environmental databases used for this study are available from J.T.K.

Environmental Measurements. A range of measurements of energy (based on productivity and climate) and habitat variability were made with high-resolution digital data. Energy-related data included PET and actual evapotranspiration (AET; ref. 22) and Chikogo NPP (23), all at 30-min resolution. The difference between PET and AET was also calculated, because this measures aridity (or water deficit) directly as a function of heat and water availability. Digital elevation data (5-min resolution; ref. 24) were used to measure topographical variation within each quadrat. The average, minimum, maximum, and range of each variable were extracted for every quadrat in each grid.

Measurements of habitat heterogeneity were derived from two land cover classifications. The first of these is based on global advanced very high-resolution radiometer (AVHRR) data from the National Oceanographic and Atmospheric Administration pathfinder land (PAL) program that has been classified by using decision tree analysis (25). These AVHRR data, composited

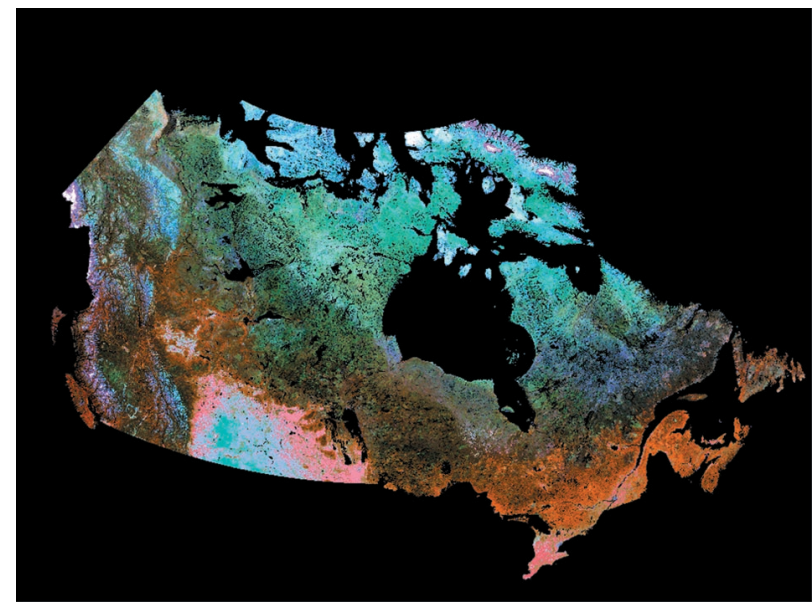

Fig. 1. High-resolution land cover map of Canada from processed classified SPOT4/VGT data (26). This is the most advanced depiction of habitat heterogeneity available for Canada. Imagery from some arctic islands was unavailable for this land cover classification.

from 1984 imagery, are relatively coarse resolution (individual pixels are about $8 \mathrm{~km} \times 8 \mathrm{~km}$ ). We also measured land cover variability from a new land cover classification by using the most recent operational sensor for large-scale observation from the Système Probatoire pour l'Observation de la Terre (SPOT), called VEGETATION (or VGT). This sensor is designed specifically for vegetation monitoring across broad geographic expanses and offers enhanced spectral and spatial characteristics (pixel size is $1 \mathrm{~km}^{2}$ ) relative to the most recent AVHRR sensor (NOAA-16). The Canada Centre for Remote Sensing and the Canadian Forest Service have produced a national-scale land cover classification, the most advanced available for the area, from the 1998 VGT composite (26). These VGT data were corrected for atmospheric interference and bidirectional reflectance effects (because the reflectance of each pixel, and its subsequent classification, is strongly affected by solar angle and satellite viewing angle) and were classified by using a new unsupervised classification method that has been applied successfully throughout Canada (ref. 13; Fig. 1). VGT imagery excluded the most northerly arctic islands in Canada, thus these were omitted from our analysis. Because the VGT land cover classification was developed largely for application in forested regions, discrimination among vegetation cover categories in such areas is enhanced relative to elsewhere. To adjust for this, some forest land cover classes were merged to major habitat classes, reducing the original land cover classification from 51 land cover types to 30 . Land cover diversity patterns, measured as the number of land cover types per quadrat, are strongly correlated between the initial and reclassed land cover products (based on $2^{\circ}$ quadrats; $R^{2}=0.946, P \ll 10^{-6}$ ). The number of vegetation classes (based on both the AVHRR and VGT classifications, respectively) in each quadrat was extracted by using ARC/INFO GRID (for VGT data) or IDRISI 32 (for AVHRR data).

Biodiversity Measurements. Butterfly species-richness information is derived from the complete Canadian National Collection (CNC) and consists of 113,525 records for 297 species (27). All butterfly records were overlaid onto each grid to calculate species-richness values on a per quadrat basis. The number of records per quadrat for all species was recorded as an index of sampling intensity, and we assessed its influence by including it in all regression models of species richness. In general, sampling intensity should be highest near the southern border of Canada and should decline toward northern Canada. The relative effect 


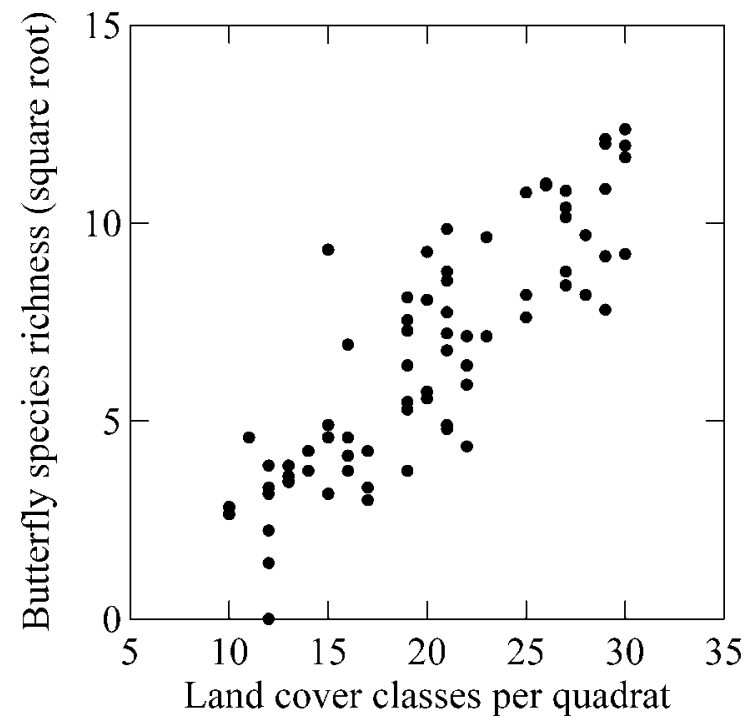

Fig. 2. The relationship between land cover diversity (derived from VGT land cover) and butterfly species richness (square root-transformed) for the $5^{\circ}$ quadrat grid. The relationship weakens as quadrat size declines but remains the strongest we could detect at any scale.

of sampling intensity on observed patterns of biodiversity can only rarely be measured at this scale as most biodiversity analyses employ range maps rather than complete lists of collection records. The CNC butterfly database is unusual because of its high collection intensity, broad geographic coverage, and because it consists of precisely georeferenced sampling locales rather than interpolated range maps.

To investigate the relationship between community similarity and environmental factors, the Jaccard index of community similarity was used to compare the butterfly species similarity and land cover similarity, respectively, along a transect through southern Canada. Sampling intensity is highest in this area. The possibility that climate influences community similarity was investigated by calculating the difference between mean PET, NPP, and actual evapotranspiration for each quadrat pair for which the Jaccard index (28) was calculated. The Jaccard index is

$$
C_{\mathrm{J}}=\frac{j}{(a+b-j)}
$$

where $j$ is the number of entities (species or land cover types) common to both quadrats, $a$ is the equivalent value for the most western quadrat alone, and $b$ is the number of species or land covers in the comparison quadrat. The index was calculated for the westernmost quadrat in the transect and compared with each successive quadrat eastwards until the transect reached the end of mainland Canada. Community similarity should decline with increasing separation between quadrats, thus separation distance was measured and "partialled" out by regressing all variables against distance and by using their residuals in further analysis (29). Adjustment for distance removes the spatial component of the variation for the variables in this analysis, essentially eliminating spatial autocorrelation (SA) from the data.

Statistical Analysis. The primary analyses in this study-of species richness and community similarity, respectively-were based on similar statistical approaches. In general, bivariate plots of diversity and environmental variables were constructed to provide estimates of the shapes of these relationships and their error distributions. Most relationships were nearly linear with only

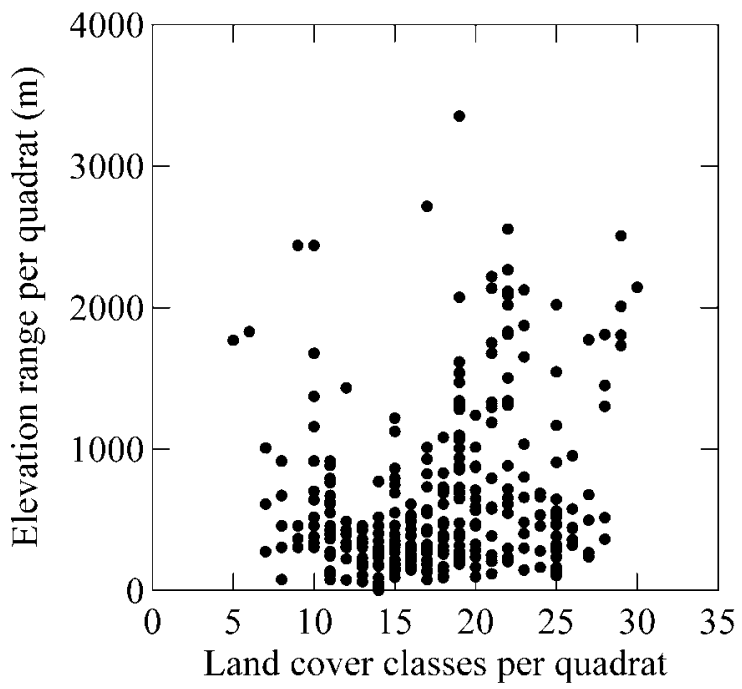

Fig. 3. The relationship between land cover diversity and elevation variability (in meters) based on $2^{\circ}$ quadrats.

minor deviations from the assumptions of standard parametric statistical analyses, namely, normal distribution and equality of variance among residuals. All transformations used to stabilize residual variance among variables in the final regression models are reported (Table 1). Main statistical analysis of relationships consisted of multiple regressions with tests for residual SA effects $(30,31)$. Forward and backward regression techniques were used to derive the "best" regression model. Variables were excluded from the final model if they did not exceed a tolerance of 0.02 or did not have an individual probability of significance $<10^{-3}$

SA weakens probability tests by reducing the effective number of degrees of freedom (32). The presence of significant SA among residuals creates spatially dependent trends that may violate standard assumptions of parametric statistical tests. SA does not render coefficients of determination or $F$ statistics invalid. The most widely used measurement of SA is Moran's I (33), which is calculated for the residuals of each regression model constructed here. This statistic, qualitatively similar to a correlation coefficient, measures the propensity for data values to covary as a function of their separation distance (or "lag"). Large absolute values of Moran's I indicate that significant spatial trend remains in the residuals, potentially causing violations of assumptions of homoscedasticity. If the regression model includes all major predictors of species-richness patterns, the residuals should be free of SA (that is, the values of the residuals should appear to be random with respect to spatial position). Rook's Case (31) was used to calculate SA statistics for the residuals of all regression equations based on the spatial distribution of data points on a Cartesian surface. Monte Carlo simulations $(n=10,000)$ based on the median first lag distance separating data points were performed to estimate significance of the Moran statistic.

\section{Results and Discussion}

The number of VGT land cover classes per quadrat was found to be the best environmental predictor of butterfly species richness at all sampling grains. The predictability of butterfly richness improved with quadrat size (i.e., coefficient of determination increased and model rms error declined; Table 1). Heterogeneity measured by coarse resolution AVHRR data and PET made comparably strong predictions of butterfly species richness. Topographic relief is poorly correlated with land cover 
Table 1. Final regression models linking patterns of butterfly species richness and community structure with environmental predictors

\begin{tabular}{|c|c|c|c|c|c|}
\hline Dependent variable & Independent variable & $\begin{array}{l}\text { Coefficient } \\
\text { (std. coeff.) }\end{array}$ & Factor $P$ & $\begin{array}{l}\text { Adj. } R^{2} \\
\text { (rms) }\end{array}$ & $\begin{array}{l}\text { Model } P \\
\quad(n)\end{array}$ \\
\hline \multirow{4}{*}{$\begin{array}{l}\text { (Butterfly species richness) })^{0.5} \\
\left(5^{\circ} \text { quadrats }\right)\end{array}$} & VGT land cover diversity & $0.221(0.514)$ & $<10^{-6}$ & 0.904 & $\ll 10^{-6}$ \\
\hline & Maximum PET & $0.00332(0.249)$ & $<10^{-5}$ & $(1.01)$ & (72) \\
\hline & Minimum elevation & $0.00030(0.239)$ & $<10^{-5}$ & & \\
\hline & Sampling & $0.00478(0.216)$ & $<10^{-5}$ & & \\
\hline \multirow{4}{*}{$\begin{array}{l}\text { (Butterfly species richness) })^{0.5} \\
\left(2.5^{\circ} \times 5^{\circ} \text { quadrats)* }\right.\end{array}$} & VGT land cover diversity & $0.217(0.390)$ & $<10^{-6}$ & 0.710 & $\ll 10^{-6}$ \\
\hline & Maximum PET & $0.00206(0.156)$ & 0.00180 & $(1.61)$ & $(161)$ \\
\hline & Minimum elevation & $0.00069(0.168)$ & 0.00040 & & \\
\hline & Sampling & $0.00012(0.392)$ & $<10^{-6}$ & & \\
\hline \multirow{3}{*}{$\begin{array}{l}\text { (Butterfly species richness })^{0.5} \\
\quad\left(2^{\circ} \text { quadrats }\right)\end{array}$} & VGT land cover diversity & $0.191(0.407)$ & $<10^{-6}$ & 0.622 & $\ll 10^{-6}$ \\
\hline & Maximum PET & $0.00386(0.231)$ & $<10^{-6}$ & $(1.96)$ & $(371)$ \\
\hline & Sampling & $0.00201(0.347)$ & $<10^{-6}$ & & \\
\hline $\begin{array}{l}\text { Jaccard values for butterflies } \\
\text { (community similarity) }\end{array}$ & $\begin{array}{l}\text { Jaccard value for VGT } \\
\text { land cover composition }\end{array}$ & 0.0891 & $<10^{-4}$ & 0.547 & $\begin{array}{c}<10^{-4} \\
(23)\end{array}$ \\
\hline
\end{tabular}

Sampling intensity should be interpreted with caution. Standardized coefficients, estimating the relative importance of each variable, are included after the regression coefficient. The rms errors of multiple regression models provide additional evidence that the predictability of butterfly richness increases with sampling grain.

*This quadrat system is included to facilitate comparison with previous diversity studies (e.g., refs. 5 and 8). Some quadrats near the southern border region of Canada are of $2.5 \times 2.5$ degrees.

diversity $\left(R^{2}=0.0781, P<10^{-5}\right.$; Fig. 3$)$, probably because it reflects habitat heterogeneity relatively poorly in Canada.

Although magnitudes of all correlations in this study decreased with quadrat size, their relative importance remained consistent (i.e., habitat heterogeneity exhibits the strongest correlations at all scales, energy the second strongest, and so on). Butterfly richness may be less predictable as quadrat size declines because of increased measurement errors associated with sampling problems. In studies based on interpolated species distributions, the reverse problem is expected: many quadrats will claim more species than are actually present. The true number of species rests in between these two estimates, but the use of primary data seems preferable when possible.

Energy-related factors improve predictions of butterfly species richness but are less important at all scales than our high-resolution measurement of habitat heterogeneity (Table 1; Fig. 4). PET does not exhibit unusually weak correlations with butterfly species richness in this study; rather, habitat heterogeneity makes unusually strong predictions. This result seems to support earlier findings that suggested that environmentdiversity relationships are scale-dependant (4) and also that habitat heterogeneity may be a more significant determinant of large-scale diversity in some regions $(3,34)$. Water deficit, measured as the difference between potential and actual evapotranspiration, was never significant in our final statistical models.

There are at least two pathways for energy to affect butterfly species richness. First, through physiological tolerance $(35,36)$ or some other mechanism (such as resource partitioning), energy availability may increase the number of species that may persist within an individual habitat (37). Second, through aggregated effects on individual species, climate helps determine what habitats may form within a region (e.g., ref. 38). The role of energy in potential and previously detected effects of climate change on biodiversity (39) should be investigated more fully in two ways: first, through its direct effects on species and species distributions through physiological tolerances and, second, through the indirect effects of climatically induced habitat changes. Biotic interactions may complicate species' responses to climate change (40) but such extraordinarily detailed ecological data are usually unavailable across broad areas. Prediction and monitoring of climate change impacts on biodiversity are unlikely to be widely successful unless relationships between richness and both habitat heterogeneity and climate are considered.
VGT land cover diversity and species richness correlate monotically but with a "shallow" slope (Fig. 2; Table 1). The slope of the relationship between species richness (square roottransformed) and land cover diversity is about 0.20 irrespective of quadrat size [test for equality of multiple slopes (29): $F=$ $0.404, P>0.5]$. There are clear conservation implications for a direct, strong link between habitat diversity and species richness across very large areas and the discovery of the consistent slope characterizing this relationship. Our results suggest that some extinctions should be anticipated after substantial declines in habitat diversity, and that preservation of habitats is a general requirement for successful conservation. Climate-based models of species richness are neutral on this issue and have an equivocal mechanistic basis. The relationship between extinction rates and habitat loss needs urgent exploration in Canada and elsewhere. Further consideration of the influence of habitat heterogeneity

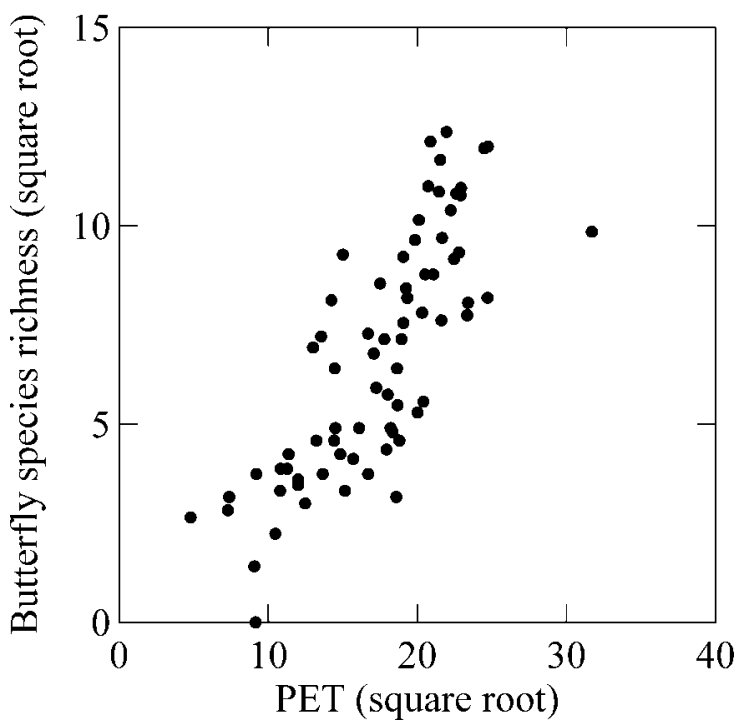

Fig. 4. The relationship between mean potential evapotranspiration (mm/ year) and butterfly species richness based on $5^{\circ}$ quadrats. Both variables are square root-transformed. The relationship, which is strongly positive at all spatial scales in this study, is always less significant than that between VGT land cover diversity and butterfly species richness. 


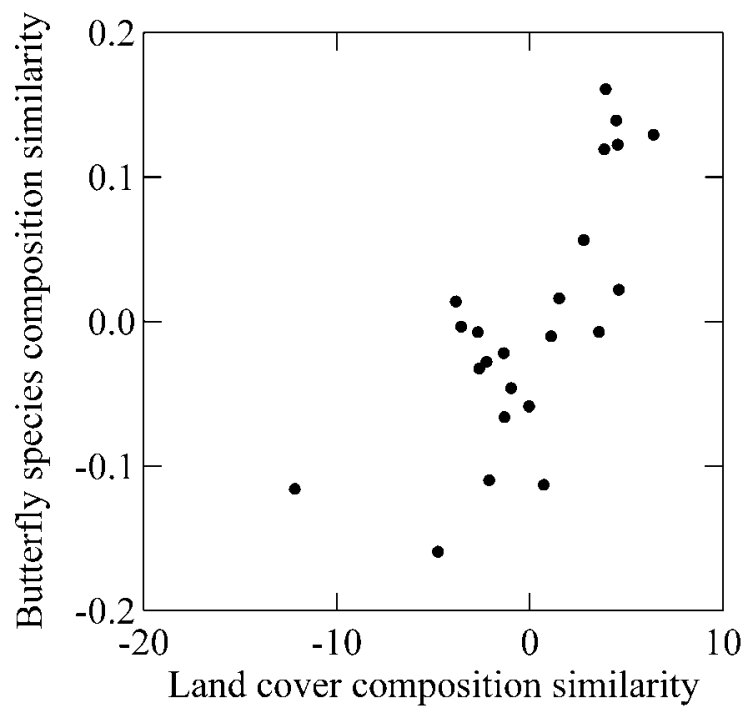

Fig. 5. The relationship between butterfly community similarity and land cover similarity based on $2.5^{\circ} \times 2.5^{\circ}$ quadrats in a transect across southern Canada. These values are distance-adjusted residuals so they are free of spatial effects. Community similarity is strongly positively related to similarity in habitat composition but unrelated to differences in energy availability.

on endemism would also be useful (e.g., refs. 41-44), as would an assessment of the threat to endemic species from habitat loss.

Similarities in land cover composition relate to butterfly community similarity in southern Canada, independent of separation distance between quadrats (Fig. 5; Table $1 ; R^{2}=0.549$, $\left.P<10^{-5}\right)$. This pattern is consistent with the hypothesis that differences in habitat composition determine differences in species composition (i.e., that species turnover increases with habitat differences). At a regional scale, this result is expected if habitat heterogeneity predicts species richness, because it is associated with greater beta diversity. On the other hand, differences in respective NPP and PET values between quadrats do not negatively correlate with community similarity as they should were they the cause of changes in biotic community composition (PET: $R^{2}=0.0686, P=0.227$; NPP: $R^{2}=0.0946$, $P=0.153)$. These climate-related factors may influence differences in biotic community composition indirectly through effects on habitats that may be present within an individual quadrat, but their role in determining differences in butterfly community structure seems to be unimportant.

Sampling intensity, measured as the number of butterfly records in each quadrat, generally improves regression models. Sampling intensity is highest in the southern areas of Canada and weakest in the high Arctic, as expected. The slope of the sampling-richness curves are low (Table 1), indicating that many records must be collected for a single additional species to be added to the quadrat total. This finding is consistent with well sampled biodiversity data (45). Although many quadrats un-

1. Currie, D. J., Kerr, J. T. \& Francis, A. (1999) Ecoscience 6, 392-399.

2. Gaston, K. J. (2000) Nature (London) 405, 220-227.

3. Kerr, J. T. \& Packer, L. (1997) Nature (London) 385, 252-254.

4. Rahbek, C. \& Graves, G. R. (2001) Proc. R. Soc. Biol. Sci. 267, 2259-2265.

5. Currie, D. J. (1991) Am. Nat. 137, 27-49.

6. Rosenzweig, M. L. (1995) Species Diversity in Space and Time (Cambridge Univ. Press, Cambridge, U.K.)

7. Guégan, J., Lek, S. \& Oberdorff, T. (1998) Nature (London) 391, 382-384.

8. Currie, D. J. \& Paquin, V. (1987) Nature (London) 329, 326-327.

9. Turner, J. R. G., Gatehouse, C. M. \& Corey, C. A. (1987) Oikos 48, 195-205.

10. Lennon, J. J., Greenwood, J. J. D. \& Turner, J. R. G. (2000) J. Anim. Ecol. 69, $581-598$ doubtedly underestimate species richness to some extent, the general diversity patterns we document resemble those for other groups. Furthermore, quadrats at relatively well collected sites in the north (e.g., near military sites, national parks, and towns) have only slightly higher diversity than less well sampled locales. In addition, the number of records in each quadrat will correlate with species richness independently of sampling intensity to some extent for two reasons. First, butterflies may be easier to catch and find in areas where their richness is high. Second, there are fewer redundant records of species (i.e., a species is recorded more than once per quadrat) in areas of low diversity because there is less of interest to the butterfly researcher there and because such areas are often remote. Any analysis of sampling intensity at this scale is likely to be similarly flawed. Biodiversity data are never as comprehensive across broad geographic regions as remote sensing data although the Canadian National Collection is one of the best of such databases.

SA is not significant at any spatial scale based on tests of spatial dependence among regression residuals. Nevertheless, significance tests are probably too liberal given nonindependence of input data points, therefore probability values should be interpreted with some caution. For all our models, the respective $F$ statistics (and coefficients of determination) are high enough that, even with only a few degrees of freedom, they would remain significant. For tests involving community similarity, the spatial component of all variables, and consequently SA, has been removed by controlling for pairwise distances between quadrats.

\section{Conclusions}

Habitat heterogeneity, as estimated by an advanced land cover classification, provides a stronger prediction of butterfly species richness in Canada than any previously measured factor. At large spatial scales, virtually all spatial variability $(>90 \%)$ in butterfly richness patterns is explained by habitat heterogeneity with secondary but significant contributions from climate (especially PET) and topography. Patterns of species turnover across the best sampled southern region of Canada are strongly related to differences in habitat composition, supporting species turnover as the mechanism through which land cover diversity may influence butterfly richness. Differences in climate are unrelated to butterfly community similarity at this scale, suggesting that the influences of energy on richness may be indirect or limited to within-habitat diversity. These results have significant conservation implications and indicate that the role of habitat heterogeneity may be considerably more important in determining large-scale species-richness patterns than previously assumed.

Larry Speers and Peter Hall made the Canadian National Collection butterfly data available for our analysis. Robert May provided scientific advice and research support. Critical comments from David Currie, Laurence Packer, Brad Hawkins, Richard Fernandes, and Simon Hay improved our manuscript. J.T.K. was supported by a postdoctoral fellowship from the Natural Sciences and Engineering Research Council of Canada while at Oxford. The Canadian Space Agency and Climate Change and Ecosystem Impacts Project provided support through the Canada Centre for Remote Sensing. T.R.E.S. is grateful for a Leverhulme Emeritus Fellowship.

11. Kerr, J. T. (1999) Biodiversity and Conservation 8, 617-628.

12. Kerr, J. T. (2001) Trends Ecol. Evol. 16, 424-425.

13. Beaubien, J., Cihlar, J., Simard, G. \& Latifovic, R. (1999) J. Geophys. Res. 104, 27909-27920

14. Cihlar, J. (2000) Int. J. Remote Sensing 21, 1093-1114.

15. Chen, J. \& Cihlar, J. (2001) in Vegetation 2000 Proceedings (Belgirate, Italy).

16. Fjeldsa, J., Lambin, E. \& Mertens, B. (1999) Ecography 21, 63-78.

17. Gascon, C., Malcolm, J. R., Patton, J. L., da Silva, M. N. F., Bogard, J. P., Lougheed, S. C., Peres, C. A., Neckel, S. \& Boag, P. T. (2000) Proc. Natl. Acad. Sci. USA 97, 13672-13677. (First Published November 28, 2000; 10.1073/ pnas.230136397)

18. Ricklef, R. E. \& Qian, H. (2000) Nature (London) 407, 180-182. 
19. Kerr, J. T., Vincent, R. \& Currie, D. J. (1998) Ecoscience 5, 448-453.

20. Wright, D. H., Currie, D. J. \& Maurer, B. A. (1993) in Species Diversity in Ecological Communities, eds. Ricklefs, R. E. \& Schluter, D. (Univ. Chicago Press, Chicago), pp. 66-76.

21. Wright, D. H. (1983) Oikos 41, 496-506.

22. Ahn, C. H. \& Tateishi, R. (1994) J. Jpn. Soc. Photogrammetry Remote Sensing 33, 12-21.

23. Uchijima, Z. \& Seino, H. (1985) J. Agric. Meteorol. 40, 343-352.

24. National Geophysical Data Center. (1988) Data Announcement 88-MGG- 02, Digital Relief of the Surface of the Earth (Natl. Oceanogr. Atmos. Admin., Boulder, Colorado).

25. Defries, R. S., Hansen, M., Townshend, J. R. G. \& Sohlberg, R. (1998) Int. J. Remote Sensing 19, 3141-3168.

26. Cihlar, J., Beaubien, J. \& Latifovic, R. (2001) VGT Land Cover of Canada (Natural Resources Canada, Ottawa).

27. Layberry, R. A., Hall, P. W. \& Lafontaine, D. J. (1998) The Butterflies of Canada (Univ. Toronto Press, Toronto).

28. Southwood, T. R. E. (2000) Ecological Methods (Blackwell Science, Oxford), 3rd. Ed.

29. Zar, J. H. (1984) Biostatistical Analysis (Prentice-Hall, Englewood Cliffs, NJ).

30. Anselin, L. (1992) Technical report S-92-1 of the National Center for Geographic Information and Analysis (Univ. California, Santa Barbara).

31. Sawada, M. (1999) Bull. Ecol. Soc. Am. 80, 231-234.
32. Koenig, W. D. (1999) Trends Ecol. Evol. 14, 22-26.

33. Sokal, R. R. \& Oden, N. L. (1978) Biol. J. Linn. Soc. 10, 199-228.

34. Rahbek, C. \& Graves, G. R. (2001) Proc. Natl. Acad. Sci. USA 98, 45344539 .

35. Kukal, O., Ayres, M. P. \& Scriber, J. M. (1991) Can. J. Zool. 69, 3028-3037.

36. Root, T. (1988) Ecology 69, 330-339.

37. Currie, D. J. \& Fritz, J. T. (1993) Oikos 67, 56-68.

38. Woodward, F. I. (1987) Climate and Plant Distribution (Cambridge Univ. Press, Cambridge, U.K.).

39. Parmesan, C., Ryrholm, N., Steganescu, C., Hill, J. K., Thomas, C. D., Descimon, H., Huntley, B., Kaila, L., Kullberg, J., Tammaru, T., et al. (1999) Nature (London) 399, 579-583.

40. Davis, A. J., Jenkinson, L. S., Lawton, J. H., Shorrocks, B. \& Wood, S. (1998) Nature (London) 391, 783-785.

41. Kerr, J. T. (1997) Cons. Biol. 11, 1094-1100.

42. Pagel, M. D., May, R. M. \& Collie, A. R. (1991) Am. Nat. 137, 791-815.

43. Pitman, N. C. A., Terborgh, J., Silman, M. R. \& Nuez, P. (1999) Ecology 80, 2651-2661.

44. Gaston, K. J., Blackburn, T. M. \& Spicer, J. I. (1998) Trends Ecol. Evol. 13, $70-74$.

45. Colwell, R. K. \& Coddington, J. A. (1994) Philos. Trans. R. Soc. London B 345 , 101-118. 